\title{
Supraventricular Tachycardia in Pregnancy
}

\author{
Prabhu Gauri $^{1}$, Shubha SR ${ }^{2}$, M.B.Bellad ${ }^{3}$, Metgud Shridevi ${ }^{2}$
}

1. Assistant Professor, Obstetrics \&Gynaecology, Department of Obstetrics \&Gynaecology

Submission : 5-1-2021

Review : 8-1-2021

Acceptance 10-1-2021

\section{Postgraduate student, Obstetrics \&Gynaecology}

3. Professor, Obstetrics \&Gynaecology

Affiliation:KLE Academy of Higher Education and Research, Jawaharlal Nehru Medical College and Karnataka Lingayat Educational Society's Dr.Prabhakar Kore Hospital \& Medical Research Centre, Belagavi, Karnataka.

DOI: 10.47799/pimr.0803.19

(C) 2020-21 Prathima Institue of Medical Sciences

\section{Abstract}

INTRODUCTION:

The commonest arrhythmia in women of reproductive age, is paroxysmal supraventricular tachycardia (SVT). We present a rare case of SVT who presented for the first time during pregnancy, who failed to respond to Electrical cardioversion(ECV), but reverted back to sinus rhythm by secondline pharmacotherapy.

\section{PROCEDURE:}

A 22 year old primigravidapresented at 37 weeks in labour with complaints of breathlessness and severe palpitations when she was diagnosed to have supraventricular tachycardia(SVT) on ECG and was referred to a tertiary care centre for further management.Pharmacological cardioversion was attempted with intravenous diltiazem, but in vain. Decision was taken for electrical cardioversion with synchronized DC shocks of 50 joules and 100 joules successively, but was not successful too. As a last resort, bolus of intravenous Amiodarone $150 \mathrm{mg}$ was given over 10 minutes followed by infusion at the rate of 24 $\mathrm{mg}$ per hour $(2 \mathrm{ml} / \mathrm{hr})$, which finally brought down the heart rate to $98 \mathrm{bpm}$. In view of non-reassuring fetal heart rate observed on CTG, patient was taken up for an emergency caesarean section under epidural anaesthesia with grave risk consent and shifted to ICCU post-operatively.

\section{RESULT:}

Patient delivered a male baby of birth weight $2.35 \mathrm{~kg}$. Patient tolerated the surgery well and did not experience any episodes of PSVT throughout the intra-operative period. Postoperatively patient was managed in consultation with cardiologist. Amiodarone infusion was continued for 24 hours at $24 \mathrm{mg} /$ hour. Post-operative period was uneventful, patient was started on oral anti arrhythmic medications and discharged on the same.

\section{CONCLUSION :}

Accurate diagnosis, regular follow up and multidisciplinary approach during acute episode and during delivery can prevent life threatening risks that might be posed to the mother and fetus in a case of PSVT. Treatment options include nonpharmacological therapy, followed by adenosine and other drugs if required, and lastly electrical cardioversion.
KEY WORDS :

- Pregnancy

- PSVT (Paroxysmal supraventricular tachycardia)

- Cardioversion

- Anti arrhythmic drugs

- ECV (Electrical Cardio Version)

\section{INTRODUCTION:}

The commonest arrhythmia in women of reproductive age, is paroxysmal supraventricular tachycardia (SVT). Paroxysmal SVT is defined as any tachyarrhythmia with a heart rate greater than 120 beats per minute. ${ }^{1}$ The term 'paroxysmal' describes an arrhythmia that begins and ends abruptly. ${ }^{1}$ An increased incidence of cardiac arrhythmias has been reported during pregnancy in patients with and without identifiable heart disease. $^{2}$ The incidencein the general population is 35 per $1,00,000$ person years. ${ }^{3}$

We present a rare case of supraventricular tachycardia presenting for the first time during pregnancy when patient was in labour, who failed to respond to ECV, but reverted back to sinus rhythm by second line pharmacotherapy.

\section{CASE REPORT}

A 22 year old primigravidapresented at 9 months of amenorrhea presented with complaints of labour pain and complaints of mild breathlessness and severe palpitations since 10hours, which was not associated with cough or sweating for which she consulted at a nearby private hospital. The patient was then referred to a secondary care centre where she was diagnosed to have supraventricular tachycardia(SVT) on ECG and was given 2 Tablets of Diltiazem 300mg and DC shock of 100 joules, even after which SVT was not reverted, hence was referred to a tertiary care centre for further management.

The patient had taken regular antenatal visits and all three trimesters remained uneventful. On admission, a pulse rate of $210 \mathrm{bpm}$ was noted, with a blood pressureof 100/60 mm $\mathrm{Hg}$.There was no history of chest pain or syncope or similar complaints at the time of admission or anytime during pregnancy or prior.Auscultation of heart revealed tachycardia. Obstetric examination revealed uterus corresponding to 34 weeks size with fetus in cephalic presentation and on cervical examination, os was $3 \mathrm{cms}$ dilated, $40 \%$ effaced with vertex at 
-1 station and membranes intact. The patient was evaluated with routine blood investigations which ruled out hyperthyroidism and anemia. Obstetric scan was done which gave an estimated fetal weight of $2.6 \mathrm{~kg}$ and a normal amniotic fluid index.

An electrocardiogram was done on an emergency basis, which revealed a pattern of supraventricular tachycardia. Opinion of cardiologist was sought for ongoing SVT. Pharmacological cardioversion was attempted with intravenous Diltiazem $10 \mathrm{mg}$ (given in 2 divided doses over every 2 minutes), but failed. Hence decision was taken for electrical cardioversion with synchronized DC shocks of 50 joules and 100 joules successively, but again was not successful in reverting the heart rate. Then a bolus of intravenous Amiodarone $150 \mathrm{mg}$ was given over 10 minutes followed by infusion at the rate of $24 \mathrm{mg}$ per hour $(2 \mathrm{ml}$ $\mathrm{hr}$ ), which finally brought down the heart rate to $98 \mathrm{bpm}$ (Fig. 1).

In view of non-reassuring fetal heart rate observed on CTG, an emergency caesarean section with grave risk consent was planned. Patient was induced with epidural anaesthesia, delivered a male baby of birth weight $2.35 \mathrm{~kg}$. Baby did not cry immediately after birth, after initial suctioning, baby received positive pressure ventilation with $A M B U$ bag for 15 secondsand by 5 minutes regular respiratory efforts were established. Intraoperatively, meconium stained liquor was noted, uterus was flabby and was managed with uterotonics and with Hayman's compression sutures. Patient tolerated the surgery well and never experienced any episodes of PSVT throughout the intra-operative period.

Patient was shifted to Intensive coronary care unit for postoperative monitoring.2D Echo was done, which showed global hypokinesia of left ventricle with mild left ventricular dysfunction with ejection fraction of $45 \%$, Grade II eccentric mitral regurgitation and Grade I tricuspid regurgitation were observed,Estimated Pulmonary Artery pressurewas $33 \mathrm{mmHg}$, Mild PAH was noted with normal Left aortic arch. As per cardiology opinion, amiodarone infusion was continued for 24 hours at $24 \mathrm{mg}$ per hour,then discontinued. Tablet diltiazem $60 \mathrm{mg}$ and tablet dytor $5 \mathrm{mg}$ were started. Along with the above, subcutaneous heparin injection was also advised for prophylactic prevention of DVT. There was no further episode of PSVT and patient maintained sinus rhythm after initial cardioversion and amiodarone injection. Epidural top-ups were administered with $8 \mathrm{ml}$ of $0.125 \%$ bupivacaine at 12 hourly intervals for $48 \mathrm{hrs}$. Patient was shifted back to labour ward on post-operative day 1 and was managed as per routine post LSCS protocol. Case was reviewed by cardiologist before discharge and as there was nil active intervention required, patient was advised to continue the ongoing treatment.

\section{Figure legends}

Figure 1: Electrocardiogram (ECG) of the patient on admission showing supraventricular tachycardia (SVT) pattern

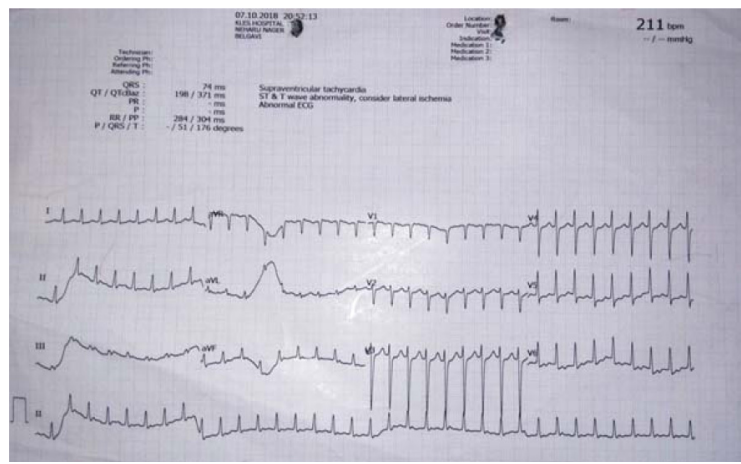

\section{DISCUSSION}

Supraventricular or ventricular tachyarrhythmias can become more frequent or may develop for the first time during pregnancy. ${ }^{4}$ The commonest Supraventricular tachycardia is Wolf-Parkinsons white syndrome and atrioventricular nodal re-entrant tachycardia, with incidence of 1.2 per 1000 people. ${ }^{5}$ The main mechanism for the development of SVT is via re-entry, most commonly atrioventricular nodal re-entrant tachycardia (in $60 \%$ of cases) and atrioventricular re-entrant tachycardia (in 30\%). ${ }^{6}$ When SVT occurs during pregnancy, women commonly present with symptoms like dizziness, palpitations, syncope, presyncope, chestpain, uneasiness and Cardiac failure. Prompt recognition and multidisciplinary approach by early involvement of cardiologist, will help in reducing maternal and perinatal morbidity and mortality.

For accurate diagnosis of arrhythmias, a proper clinical examination aided by a 12 - lead ECG is necessary. ${ }^{1,7}$ Also, an echocardiography is essential so as to exclude any structural or functional heart diseases as their presence may pose a threat for development of arrhythmias during pregnancy. Hence for accurate diagnosis and management of such cases, a multidisciplinary approach with early involvement of cardiologist is very essential. Also it is necessary to rule out, other disorders likethyroid dysfunction, electrolyte imbalance, anemia, fever, anxiety, toxic drug use and thromboembolism, before the diagnosis of PSVT is made.

The management of acute PSVT in pregnancy is started with non pharmacological manoeuvre which includes vagal stimulation by carotid massage and Valsalva manoeuvre with simultaneous positioning of the patient in left lateral position. If the above mentioned procedures fail to revert SVT, then the drug of choice is adenosine, followed by low dose of b-blockers. Adenosine is the preferred first line drug due to its speed of action, efficacy and safety profile. ${ }^{8}$ Second choice is verapamil which is a calcium channel blocker, but only after the first trimester of pregnancy and only in acute circumstances. It is the alternative if the tachycardia is not reverted or in asthamatic women. ${ }^{8}$ DC cardioversion is safe in all phases of 
pregnancy and should be considered in women who are refractory for pharmacotherapy and who are hemodynamically unstable.There is considerable variation in required energy varied from 50 to $400 \mathrm{~J}$. During acute management of PSVT by cardioverison, continuous electronic fetal monitoring is advisable to reduce risk of fetalarrhythmogenesis from electrical discharge.

Hence to conclude, accurate diagnosis, regular follow up and multidisciplinary approach during acute episode and during delivery can prevent life threatening risks that might be posed to the mother and fetus. Non pharmacological therapy, followed by adenosine and other drugs if required, and lastly ECV with adequate maternal and fetal monitoring, can be used with good success rates in intractable cases.

\section{REFERENCES}

1. Robins K, Lyons G. Supraventricular tachycardia in pregnancy. Br J Anaesth 2004;92(1):140-143.

2. Shotan A, Ostrzega E, Mehra A, et al., Incidence of arrhythmias in normal pregnancy and relation to palpitations, dizziness, and syncope, Am J Cardiol, 1997;79:1061-4.

3. Orejarena LA, Vidaillet H Jr, DeStefano F, et al. Paroxysmal supraventricular tachycardia in the general population. J Am CollCardiol. 1998;31(1):150-7.

4. Tan HL, Lie KI, Treatment of tachyarrhythmias during pregnancy and lactation, Eur Heart J, 2001;22:458-64.

5. Rotmensch HH, Rotmensch S, Elkkayam U. Management of cardiac arrythmias during pregnancy. Current concepts. Drugs 1987;33(6):623-33

6. Ghosh N, Luk A, Derzko C, et al. The acute treatment of maternal supraventricular tachycardias during pregnancy: a review of the literature. J ObstetGynaecol Can. 2011;33(1):17-23.

7. Tromp CH, Nanne AC, Pernet PJ, et al. Electrical cardioversion during pregnancy: safe or not? Neth Heart J. 2011;19(3):134-6. PubMed PMID: 21475392. Pubmed Central PMCID: 3047673

8. Carlos DPV, Diego PD. Arrhythmias in pregnancy. Emergencias 2013;25(5):397-408

How to cite this article : Prabhu G, Shubha SR, M.B.Bellad, Metgud S. Supraventricular Tachycardia in Pregnancy. Perspectives in Medical Research 2020; 8 (3):88-90.

DOI: 10.47799/pimr.0803.19

Sources of Support: Nil, Conflict of interest: None declared 